\title{
Drei Rückfragen an die normativen Grundlagen der Ordonomik
}

\section{Kommentar zum Hauptbeitrag von Ingo Pies}

[1] Der ordonomische Ansatz, wie er sich aus der ökonomischen Ethik entwickelt hat, stellt ein zunehmend beeindruckendes Theorieprogramm dar. Er geht inzwischen über eine Wirtschaftsethik, sogar eine Wirtschaftsphilosophie hinaus und nimmt Züge einer umfassenden Gesellschaftstheorie an, für die eine Auseinandersetzung mit und Lösung von normativen Koordinationsproblemen im Zentrum steht (vgl. [68]). Zu diesem Programm leisten die Arbeiten von Ingo Pies einen wesentlichen Beitrag. Mein Anliegen in diesem kurzen Kommentar zu dem vorliegenden Aufsatz von Pies soll nicht darin bestehen, die Ordonomik in ihren Grundlagen in Frage zu stellen. Ich glaube tatsächlich, dass der ordonomische Ansatz einen wichtigen Beitrag zur praxisorientierten Analyse gesellschaftlicher Probleme leisten kann. Allerdings habe ich den Eindruck, dass er sich besonders für einige Probleme in spezifischen Kontexten eignet. Vor diesem Hintergrund möchte ich mit Blick auf den Aufsatz von Pies drei Punkte herausarbeiten, in denen es meiner Ansicht nach weiteren Klärungsbedarf gibt. Das beinhaltet vor allem die Abgrenzung gegen alternative Ansätze und die Offenlegung normativer Grundlagen.

[2] Der erste Punkt betrifft die Abgrenzung gegenüber der Individualethik, die häufig, so auch in diesem Aufsatz einen ablehnenden Tonfall annimmt (vgl. [5]). Selbst wenn man der Argumentation folgt, dass sich eine bloße Individualethik nicht zur Lösung struktureller Gesellschaftsprobleme eignet, so bedeutet das nicht, dass individualethische Überlegungen irrelevant seien. Individualethikern geht es häufig um die Reflexion über das richtige oder gute Handeln einzelner Menschen in gegebenen Strukturen. Dabei soll ausgelotet werden, welche Handlungen verboten, erlaubt und vielleicht wünschenswert oder geboten sind. Viele Menschen haben ein Interesse daran, ein moralisch gutes Leben zu leben. Für diese Menschen sind individualethische Überlegungen durchaus informativ. Dabei ist es übrigens möglich, die Unvollkommenheit gegebener gesellschaftlicher Strukturen in die normativen Überlegungen mitaufzunehmen. Es gibt einen anhaltenden Streit darüber, in welchem Umfang das geschehen muss. Unter Anhängern der Position, dass eine Hilfspflicht absolut armen Menschen gegenüber besteht, gibt es beispielsweise einen Streit über das Ausmaß dieser Pflicht und ob die Inaktivität und Unvollkommenheit politischer Institutionen darauf einen Einfluss haben

* Prof. Dr. Christian Neuhäuser, Institut für Philosophie und Politikwissenschaft, Technische Universität Dortmund, Emil-Figge-Str. 50, D-44227 Dortmund, Tel.: +49(0)-231-7557912, christian.neuhaeuser@tu-dortmund.de, Forschungsschwerpunkte: Theorien der Würde, Theorien der Verantwortung, Theorien des Eigentums, Wirtschaftsphilosophie, Philosophie der internationalen Politik. 
(vgl. Wenar 2016; Singer 2016). Es ist übrigens nicht angemessen auf diese Verteidigung der Individualethik zu antworten, dass eine ökonomisch kluge Entwicklungspolitik viel effektiver sei als individuelle Hilfe. Das ist unbestritten. Aber Menschen wollen auch wissen, was ihre moralischen Pflichten sind, solange diese Entwicklungspolitik ihr volles Potential nicht entfaltet hat und absolute Armut nicht flächendeckend beseitigt ist. Es ist ebenfalls nicht klar, dass sich individuelle Pflichten unter diesen Bedingungen auf das politische Einfordern der entsprechenden Verantwortung von Staaten beschränkt. Ob das so ist oder nicht, bleibt selbst ein Teil der individualethischen Diskussion. Dasselbe gilt für andere Fragen der Politik und Individualmoral. Die Ordonomik müsste sich hier meiner Einschätzung nach entweder positionieren oder einfach anerkennen, dass es sich dabei um legitime moralische Fragen handelt, für die sie nicht zuständig ist.

[3] Es gibt noch eine zweite Funktion der Individualethik, die nicht vernachlässigt werden sollte. Auch wenn individualethische Überlegungen und individuelles Handeln allein keinen strukturellen Wandel bringen, so tragen sie dennoch zum öffentlichen Diskurs bei. Das kann disruptiv, es kann aber auch konstruktiv und beides kann abhängig von der normativen Sicht auf die Dinge erwünscht sein, weil beides zum Aufbau neuer funktionaler Strukturen und Institutionen beitragen kann (vgl. Waldron 1999; Young 1990; 2010). Individualethische Überlegungen können den öffentlichen Diskurs beeinflussen, weil sie bestimmte normative Prinzipien als gültig offenlegen oder als ungültig zurückweisen können. Häufig wird dadurch ein Raum des vernünftigen Pluralismus abgesteckt. Wenn der öffentliche Diskurs vernünftig sein soll, wären die Ergebnisse der ethischen Diskussion entsprechend zu berücksichtigen. Man denke hier beispielsweise an die Argumente der Friedens- oder Umweltbewegung. Zudem kann individualethisches Handeln den öffentlichen Diskurs beeinflussen, weil es deutlich zeigt, welche Handlungsspielräume tatsächlich vorliegen. Dadurch werden Unmöglichkeitsargumente als Schutzbehauptungen entlarvt. Hierfür stehen beispielsweise die Bewegung der Abolitionisten in Großbritannien oder die schwarze Bürgerbewegung in den USA. Natürlich können solche auf eine Veränderung der normativen Hintergrundstruktur abstellende Diskurse auch versagen. Strukturelle Lösungen werden dann nicht möglich, solange man sich nicht auf gemeinsame oder zumindest kongruente Interessen besinnt, wie Pies zurecht argumentiert. Das ändert jedoch nichts daran, dass die Individualethik die genannte begrenzte Funktion neben einer und in Bezug auf eine Ordnungsethik besitzen kann.

[4] Der zweite Punkt baut auf dieser Beobachtung auf und betrifft die Anlehnung an Hobbes. Ich akzeptiere, dass es Pies nicht um die Frage geht, was die beste Hobbes-Interpretation ist (vgl. [22]). Allerdings wird Hobbes in der philosophischen Ideengeschichte stark für seine individualistisch atomistische Sozialtheorie kritisiert. Menschen sind bei Hobbes vereinzelte Individuen ohne traditionelle oder wertbasierte Bindungen, die sich nur durch freiwillige Verträge oder Zwang zusammenschließen können (vgl. Macpherson 1973 [1962]). Durch die starke Orientierung an Hobbes scheint der ordonomische Ansatz diese Perspektive zu übernehmen oder er müsste sich davon abgrenzen und zeigen, warum ein Bezug 
auf Hobbes dann trotzdem möglich bleibt. Beides erscheint mir vor dem Hintergrund der verbreiteten Kritik begründungsbedürftig.

[5] Das zeigt sich deutlich an der unmittelbaren Bezugnahme auf Interessen. Ähnlich wie Hobbes es tut, scheint der Ansatz davon auszugehen, dass Interessen gegeben und unveränderlich sind. Allerdings bezieht sich Hobbes dabei auf das Interesse der Selbsterhaltung, was selbst ohne mechanistische Erkenntnistheorie eine gewisse Anfangsplausibilität besitzt. Viele andere und darüber hinausgehende Interessen sind hingegen überhaupt nicht gegeben und unveränderlich, sondern entstehen in sozialen Prozessen und sind durch sie revidierbar. Politische Koordinationsprobleme sind also nicht nur dadurch zu lösen, dass im Diskurs nach Alternativen gesucht wird, die die Präferenzerfüllung verschiedener gesellschaftlicher Gruppen möglichst stark befördern (vgl. [42]-[43]). Vielmehr dient der Diskurs auch dazu, andere Gruppen dazu zu bringen, ihre interessenbasierten politischen Überzeugungen zu revidieren, indem sie ihre Interessen überdenken. Dies kann kooperativ durch öffentlichen Vernunftgebrauch geschehen, bei dem es darum geht, mithilfe besserer Argumente zu überzeugen. Dies kann aber auch stärker agonal geschehen, indem bestimmte Interessen performativ als egoistisch, ungerecht, manipulativ etc. entlarvt werden. Beides kann die Wirkung haben, dass sich politische Machtverhältnisse ändern und sich dadurch eine weitere Option eröffnet, die je nach Standpunkt vernünftiger erscheint. Warum soll das grundsätzlich und nicht nur aus strategischen Gründen dann, wenn es kontraproduktiv ist, abzulehnen sein?

[6] Den Kern dieser Problematik sieht man deutlich an dem von Pies angeführten Beispiel der Flüchtlingspolitik. Seiner Ansicht nach lässt sich die von ihm diagnostizierte diskursive Blockade nur aufbrechen, wenn man in Europa gleichzeitig mehr humanitäre Hilfe in anderen Ländern leistet und die eigenen Grenzen stärker schließt (vgl. [55]). Wenn jemand jedoch davon überzeugt ist, dass es eine starke Pflicht gibt, Menschen im eigenen Land aufzunehmen oder dass sogar ein globales Recht auf Bewegungsfreiheit existiert, dann wird sie diese Position in die öffentliche Diskussion einbringen wollen (vgl. Carens 2015; Cassee 2016). Je mehr Menschen das überzeugt, desto mehr Menschen unterstützen auch eine entsprechende Politik. Die kollektive Interessenlage ändert sich radikal und die Aufnahmebereitschaft des Landes steigt. Der Ansatz von Pies basiert demgegenüber darauf, dass er die Interessenlage verschiedener sozialer Gruppen zumindest für die Zwecke der Politikempfehlung als gegeben und unveränderlich annimmt. Manchmal kann das aus pragmatischen und realistischen Gründen genau der richtige Ansatz sein. Manchmal ist das jedoch nicht der Fall. Was mir hier fehlt, ist eine Hintergrundtheorie, die dabei hilft, darüber zu entscheiden, wann was zutrifft.

[7] Der dritte Punkt, den ich mit Blick auf den ordonomischen Ansatz zur Diskussion stellen möchte, betrifft seine offensichtlich konsequentialistische Ausrichtung. Meine Rückfrage bezieht sich nicht auf den Grundlagenstreit zwischen deontologischen und konsequentialistischen Ansätzen, zu dem sich der ordonomische Ansatz allerdings auch verhalten müsste. Mir geht es hier um die Beschaffenheit der konsequentialistischen Grundlagen der Theorie. Amartya Sen hat eine 
Form von Konsequentialismus vorgeschlagen, die akteursrelative Handlungsgründe und insbesondere Rechte berücksichtigen kann (vgl. Sen 1977; 1982). Das erscheint mir sehr attraktiv. Auf den ersten Blick ist nicht klar, dass dies beim ordonomischen Ansatz auch der Fall ist oder ob er eher auf einem unmittelbaren Utilitarismus aufsetzt. Pies führt den strengen Pazifismus als Beispiel für die Verwechslung von Zielen und Mitteln an (vgl. [37]). Das Beispiel ist geschickt gewählt, andere sind vielleicht weniger eindeutig. Aber selbst wenn man zugesteht, dass es hier um Frieden als Ziel geht, spielt es jedoch eine wichtige Rolle, welche Mittel angewendet werden, um dieses Ziel zu erreichen. Unter normalen Bedingungen sind manche Mittel erlaubt, andere verboten. Ein Grund dafür könnte gerade sein, dass bestimmte Mittel grundlegende Rechte und die Würde der Menschen verletzen, so ist es etwa bei Folter oder dem Einsatz von ABC-Waffen.

[8] Letztlich scheint es von den spezifischen Zielen und davon abzuhängen, was auf dem Spiel steht, welche Mittel erlaubt sind und welche nicht. Selbst Max Weber hat zugestanden, dass man manchmal seine durchaus wertvollen Ziele nicht erreichen kann, weil die dazu nötigen Mittel inakzeptabel sind (vgl. Weber 1988 [1919]). Mir ist nicht ganz klar, wo der ordonomische Ansatz die Ressourcen hernimmt, um in dieser Frage zu entscheiden. Die Flüchtlingshilfe auf arme Nachbarländer von Krisengebieten zu konzentrieren, kann beispielsweise dazu führen, dass man sehr viele Menschen an den eigenen Grenzen abweisen muss. Wie jedoch vor allem das Verhalten der ungarischen Regierung insbesondere im Jahre 2015 gezeigt hat, geht das schnell mit starken Demütigungen einher. Ist das dann gerechtfertigt? Pies schreibt, dass man die Mittelmeerroute schließen soll (vgl. [55]). Was jedoch, wenn die dazu nötigen Mittel inakzeptabel sind? Dazu schweigt er. Es kann auch sein, dass man faule Kompromisse (vgl. Margalit 2011) mit autokratischen Ländern schließen muss, die man vielleicht nicht schließen sollte, wie beispielsweise mit der Türkei. Dies führt zu einer Ermächtigung des autokratischen Systems und zerstört die moralische Glaubwürdigkeit Europas. Wieder gilt, dass nicht klar ist, wie diese Effekte normativ gegeneinander abzuwägen sind und welche Ressourcen der Ansatz von Pies hat, um diese Fragen zu klären.

[9] Wenn Pies antwortet, dass der ordonomische Ansatz ja darauf abstellt, dass diese Probleme nicht mehr auftreten, dann überzeugt mich das nicht vollständig. Es klingt einfach viel zu utopisch, dass es in allen Fällen die Möglichkeit gibt, eine übergeordnete Lösung zu finden, die für alle an einem Konflikt beteiligten Parteien vor dem Hintergrund gegebener Interessen hinreichend zufriedenstellend sind. Das gilt insbesondere dann, wenn man die normativen Standpunkte der Akteure ernsthaft berücksichtigen will und nicht auf bloße Präferenzen reduziert (vgl. Hausmann 2012). Aus dieser Sicht kann es manchmal besser sein, bestimmte Kompromisse nicht einzugehen oder die Interessen anderer Akteure diskursiv zu bearbeiten. An dieser Stelle scheint es mir übrigens grundfalsch zu sein und der Ideengeschichte der Moralphilosophie grundlegend zu widersprechen, solch einen öffentlichen Diskurs als heteronom zu bezeichnen und das unreflektierte Festhalten an gegebenen Interessen als autonom zu adeln (vgl. [71]). Das wirkt wie bloße 
Rhetorik, denn solange die Revision der eigenen Interessen auf vernünftiger Einsicht beruht, ist sie der klassischen Sicht nach der höchste Ausdruck von Autonomie. Man ist dann zusammen mit anderen vernünftigen Akteuren ein Gesetzgeber im Reich der Zwecke, wie Kant sagen würde. Diese Einsicht der Aufklärung sollte man vielleicht nicht einfach so über Bord werfen. Zumindest müsste die Ordonomik sehr viel mehr dazu sagen, auf welcher normativen Grundlage sie sich in dieser sehr grundsätzlichen philosophischen Frage so positioniert, wie sie es tut. Das halte ich für schwierig. Deswegen erscheint es mir besser, den ordonomischen Ansatz auf diejenigen Fälle zu konzentrieren, in denen der vernünftige moralische Diskurs offensichtlich scheitert. Davon gibt es ja, und da hat Pies offensichtlich recht, mehr als genug.

\section{Literaturverzeichnis}

Carens, J. (2015): The Ethics of Immigration, Oxford: Oxford University Press.

Cassee, A. (2016): Globale Bewegungsfreiheit: Ein philosophisches Plädoyer für offene Grenzen, Berlin: Suhrkamp.

Hausman, D. (2012): Preference, Value, Choice, and Welfare, New York: Cambridge University Press.

Macpherson, C.B. (1973 [1962]): Die politische Theorie des Besitzindividualismus, Frankfurt a. M.: Suhrkamp.

Margalit, A. (2011): Über Kompromisse - und faule Kompromisse, Berlin: Suhrkamp.

Sen, A. (1977): Rational Fools: A Critique of the Behavioral Foundations of Economic Theory, in: Philosophy and Public Affairs Vol. 6/No. 4, 317-344.

Sen, A. (1982): Rights and Agency, in: Philosophy and Public Affairs Vol. 11/No. 1, 3-39.

Singer, P. (2016): Effektiver Altruismus. Eine Anleitung zum ethischen Leben, Berlin: Suhrkamp.

Waldron, J. (1999): Law and Disagreement, Oxford: Oxford University Press.

Weber, M. (1988 [1919]): Politik als Beruf, in: ders.: Gesammelte Politische Schriften, Tübingen: Mohr Siebeck, 505-560.

Wenar, L. (2016): Blood Oil, Tyrants, Violence, and the Rules That Run the World, Oxford: Oxford University Press.

Young, I. (1990): Justice and the Politics of Difference, Princeton/NJ: Princeton University Press.

Young, I. (2011): Responsibility for Justice, Oxford: Oxford University Press. 